REVISTA INTERNACIONAL DE CIENCIAS DEL DEPORTE International Journal of Sport Science

Rev. int. cienc. deporte

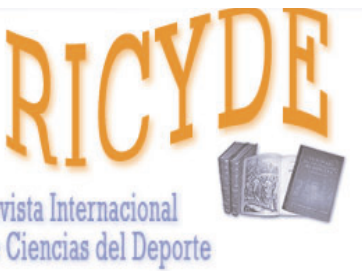

International Journal of Sport Science VOLUMEN X - AÑO X

Páginas:109-122 ISSN:1885-3137 No 36 - Abril - 2014

\title{
Uso estratégico del espacio en categorías de formación de pelota vasca Strategic use of space in basque pelota training categories
}

\author{
Oidui Usabiaga y Julen Castellano
}

Universidad del País Vasco UPV/EHU

\begin{abstract}
Resumen
La pelota vasca es un deporte de pelota compuesto por varias especialidades. La pelota a mano es una de las más practicadas y se juega tanto en duelos individuales como por equipos. El presente estudio pretende analizar y comparar algunos indicadores de juego del uso estratégico del espacio por parte de jugadores benjamines ( $8-10$ años) y alevines (10-12 años) que participan en un programa de deporte escolar. La muestra estuvo compuesta por doce partidos, seis de benjamines (30 participantes) y seis de alevines (30 participantes). El registro se llevó a cabo mediante un instrumento observacional ad hoc diseñado para analizar el uso estratégico del espacio por parte de los participantes, tanto cuando golpean la pelota como cuando están en situación de espera. Los resultados muestran que los participantes de las dos categorías usan el espacio de la misma forma respecto a las zonas de golpe, la ubicación espacial en situación de espera durante los duelos por parejas y los tipos de saque. Los alevines, en comparación a los benjamines, se posicionan más a la derecha del jugador que golpea durante los duelos individuales, dirigen la pelota a distancias medias y cometen menos faltas tanto en el resto del saque como en el juego de intercambio, dando mayor continuidad al juego $(p<0,05)$.
\end{abstract}

Palabras clave: deportes de pelota; formación; análisis notacional; espacio.

\section{Abstract}

The basque pelota is a ball sport composed of various specialties. The hand pelota is one of the most popular and is played in both individual and team matchups. The aim of this study was to analyze and compare some indicators of the strategic use of space by young players, 8-10- and 10-12-year-old students, participating in a school sport program. The sample consisted of twelve games, six for each level (30 participants for each level). Data were recorded with an ad hoc observational instrument designed to analyze the strategic use of space by the participants, both when they hit the ball and when they are on standby. The results show that the participants of the two categories used the space in the same way over hit areas, the spatial location on standby during the duels in pairs and types of serve. The 10-12-yeard-old students, compared to the youngest, are positioned to the right of the player who hits during individual duels, sent the ball at medium distances and make fewer errors in the rest and exchange beatings, giving greater continuity the game $(p<0,05)$.

Key words: ball sports; formation; notational analysis; space.

Correspondencia/correspondence: Oidui Usabiaga

Universidad del País Vasco UPV/EHU. España

Email: oidui.usabiaga@ehu.es 


\section{Introducción}

L os deportes de pelota pueden ser duelos individuales o colectivos donde los jugadores de bandos contrarios están influenciados por las conductas motrices estratégicas que despliegan los adversarios, pudiendo los jugadores reaccionar de diferente manera ante los mismos efectos de oposición (O’Donoghue, 2009). En este tipo de deportes la constante codificación del juego propio (a través de fintas) y descodificación de las conductas motrices de los adversarios desencadena un proceso de metacomunicación (Parlebas, 2001) en los jugadores, donde información y acción se entremezclan en el contexto de incertidumbre social o interacción que caracteriza esta práctica deportiva (Araujo, 2007).

Clasificado dentro de los deportes de pelota y muro, la pelota a mano en edad escolar es una especialidad de pelota vasca donde los participantes deben golpear la pelota con la "mano desnuda" y de forma alterna hacia el frontis (pared frontal) y la cancha, intentando que los adversarios no lleguen a devolverla a dichos espacios efectivos. Respecto a las reglas de juego (Federación Guipuzcoana de Pelota Vasca, 2008), en cada enfrentamiento se juegan mini-partidos donde se combinan duelos por parejas e individuales, y todos los participantes de ambos equipos pasan por los diferentes roles (sacador, restador y jugador de intercambio) de juego. Los benjamines y alevines de Gipuzkoa compiten bajo el mismo reglamento de juego, excepto el número de juegos o mini-partidos que deben disputar (los benjamines deben jugar siete mini-partidos y los alevines deben ganar cuatro juegos de siete), la distancia del saque (los benjamines deben botar la pelota en el suelo a una distancia superior a siete metros de la pared frontal y superar la línea de 2 metros de altura que está marcada en la pared frontal; los alevines deben botar la pelota en el suelo a una distancia superior a 7 metros de la pared frontal y superar la misma línea) y el diámetro y peso de la pelota (mayor diámetro y peso la pelota de alevines), que son diferentes para cada categoría.

El análisis de rendimiento se puede realizar a partir de diversos métodos que de manera sistemática permita el registro de datos del rendimiento deportivo de jugadores y equipo para ser analizados (O'Donoghue, 2010). Desde una vertiente no invasiva, el análisis notacional, ha tenido un gran desarrollo asociado a los avances tecnológicos en el ámbito de los deportes de raqueta (Hughes, Hughes, y Behan, 2007 y 2009; Vuckovic y col., 2013). Hughes (1998) y Hughes y Franks (2008) identifican diversos objetivos del análisis notacional, como la evaluación técnica o táctica, o el uso educativo que tiene dicho análisis para el educador/entrenador o el propio jugador. El uso estratégico del espacio por parte de los equipos es uno de los aspectos que está directamente relacionado con la comunicación (codificación) y contracomunicación (descodificación) entre los participantes de este tipo de deportes de adversario, y ha sido estudiado en numerosos trabajos aplicados en deportes de raqueta y muro de rendimiento, elaborando diferentes herramientas observacionales ad hoc que reúnen criterios específicos para el registro de este tipo de comportamientos (p. e., Alonso, 2004; Cabello, 2000; Garay, 2003; Gorospe, 1999; Ruiz, 1996; Usabiaga, 2005). Diferentes autores afirman que la utilización estratégica del espacio podría ser un buen indicador del rendimiento de los jugadores expertos de deportes de raqueta (Alonso y Argudo, 2007; Hughes y Barlett, 2002; O'Donogue, 2004), refiriéndose concretamente a la dirección y eficacia del servicio o saque y del resto al saque (p. e. Alonso y Argudo, 2002; Alonso, y Argudo, 2011; Gillet, Leroy, Thouvarecq, y Stein, 2009; Unierzyski y Wieczorek, 2004) o a los desplazamientos como la subida a red de tenis durante la fase de espera (p. e. Brown y O'Donoghue, 2008). 
Se han encontrado pocos trabajos que evalúan la acción de juego de participantes en etapa formativa y la mayoría de los mismos se han llevado a cabo comparando su juego con el de los expertos (Blomqvist, Luhtanen, y Laakso, 2000) o el servicio y resto al servicio en función del género (Hizan, Whipp, y Reid, 2011). El análisis de juegos y deportes tradicionales puede ser de gran ayuda para llevar a cabo las modificaciones necesarias para su adecuada aplicación en el ámbito formativo (Méndez-Giménez y Fernández-Río, 2011). No se han encontrado estudios sobre el uso estratégico del espacio en situaciones reales de juego, como indicador de rendimiento, que analizasen la acción de juego de jugadores en etapa formativa de forma contextualizada (se tiene en cuenta en el mismo multievento las conductas de golpeo y las de situación de espera) y secuencial (registro consecutivo de todas las secuencias de golpe). Esta ausencia de estudios sistémicos de la acción de juego de diferentes juegos deportivos escolares deja abierto un espacio sin cubrir.

Se partió de la hipótesis que el análisis de juego de la pelota vasca en etapas de formación podría aportar información relevante para conocer si el uso estratégico del espacio de los escolares se enriquece según cambian de categoría. Por lo tanto, la presente investigación tiene por objetivo analizar y comparar el uso estratégico del espacio durante el momento del golpeo y en situación de espera de los escolares benjamines y alevines que practican pelota a mano dentro del programa de deporte escolar de Gipuzkoa. Su aplicación en el ámbito de la intervención permitiría a los técnicos desarrollar estrategias adecuadas para optimizar las respuestas motrices dadas por los jugadores.

\section{Material y métodos}

\section{Participantes}

La muestra estudiada estuvo compuesta por 60 escolares, 30 benjamines (8-10 años) y 30 alevines (10-12 años) que participaron en doce partidos, escogidos al azar, del programa de deporte escolar de la Diputación Foral de Gipuzkoa, en el itinerario de participación. El estudio siguió las pautas establecidas en la Declaración de Helsinki. Además, todos los clubes y escuelas de pelota adscritos a la Federación Guipuzcoana de Pelota Vasca, así como los participantes y sus padres, fueron informados acerca de los objetivos del estudio y dieron su consentimiento para llevarlo a cabo.

\section{Instrumentos}

Los partidos fueron grabados con una cámara digital de disco duro de 60 gigabytes (JVC Everio GZ-HD7) que almacena las grabaciones en formato .mod. La codificación se llevó a cabo a través de la herramienta observacional EBSIS.e (Usabiaga y Castellano, 2011), diseñada para el análisis de la acción de juego de los participantes de pelota a mano en edad escolar, y que está configurado por una combinación de formatos de campo y sistemas de categorías. Con la herramienta EBSIS.e se registran secuencias de multieventos (Bakeman y Quera, 1996), donde se incluyen las siguientes variables:

- Zonas del espacio de juego donde golpean la pelota todos los participantes del juego (Figura 1), especificando la proximidad respecto al frontis y la ubicación respecto a la pared izquierda.

- Tipo de golpe empleado por el jugador que golpea la pelota, concretando la mano ejecutora y si realiza el golpe antes o después de que la pelota bote en el suelo. 
- Ubicaciones espaciales de los dos jugadores (duelos por parejas) o del adversario (duelos individuales), puntualizando si están a la izquierda, derecha o a los dos lados (duelos por parejas) del jugador que golpea la pelota.

- Dirección del golpe realizado durante el saque (primer golpe de cada tanto o punto), el resto (segundo golpe) o el juego de intercambio, registrándose la trayectoria de la pelota en referencia a la distancia respecto al frontis, la orientación de la pelota sobre el jugador que golpea (izquierda o derecha), anotando los golpes que son falta (perder el tanto) o pasa (dispone de otro saque).

La herramienta de observación EBSIS.e ha mostrado tener una alta fiabilidad (Usabiaga, Castellano, Blanco-Villaseñor, y Casamichana, 2013), siempre y cuando se aplica a partir de una cierta formación de los observadores (Anguera y Blanco-Villaseñor, 2003; Ureña, 2003).

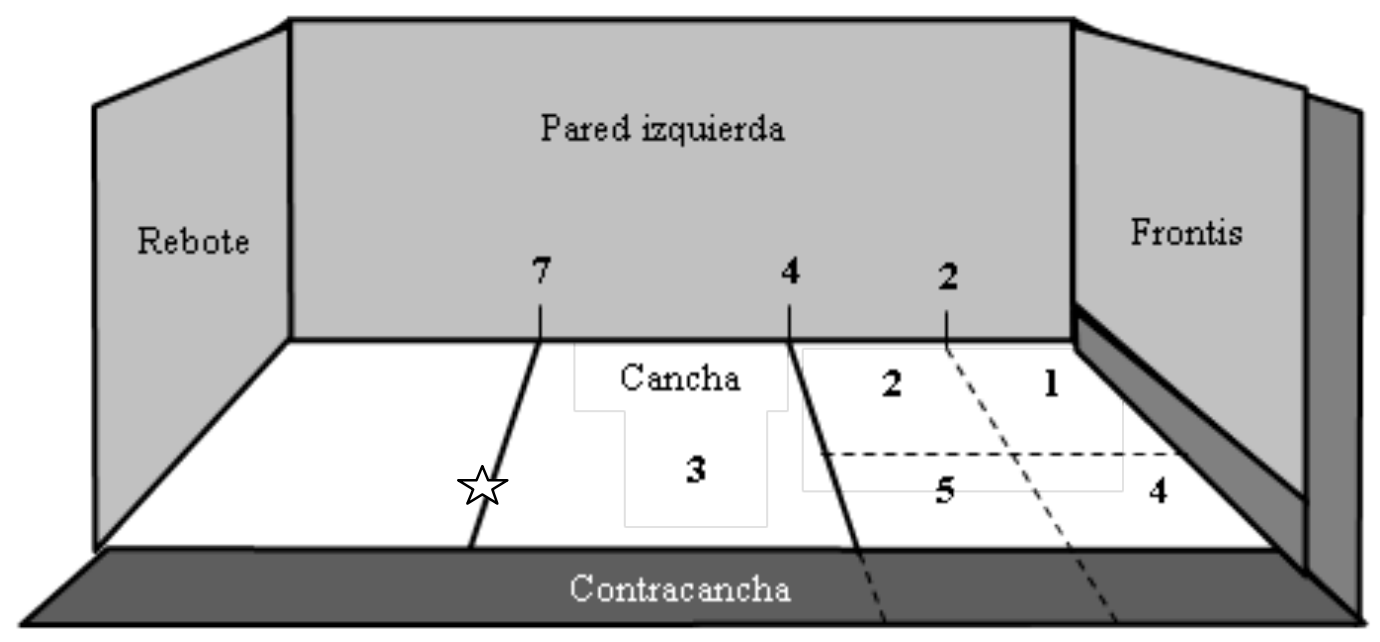

Figura 1. Representación de las categorías del criterio cartografía del espacio y ubicación espacial de la cámara de vídeo $(\stackrel{\sim}{)}$ ).

Mediante el uso del software MOTS (Castellano, Perea, Alday, y Hernández-Mendo, 2008) se registraron las conductas estratégicas de los jugadores (Figura 2). El programa SAGT v1.0 versión 211 (Ramos, Hernández-Mendo, Pastrana, y Blanco-Villaseñor, 2012) se utilizó para el análisis de la generalizabilidad o estudio G. Para la comparación de medias se empleó el paquete estadístico SPSS v.19.0 (SPSS Inc., Chicago Illinois, USA). 


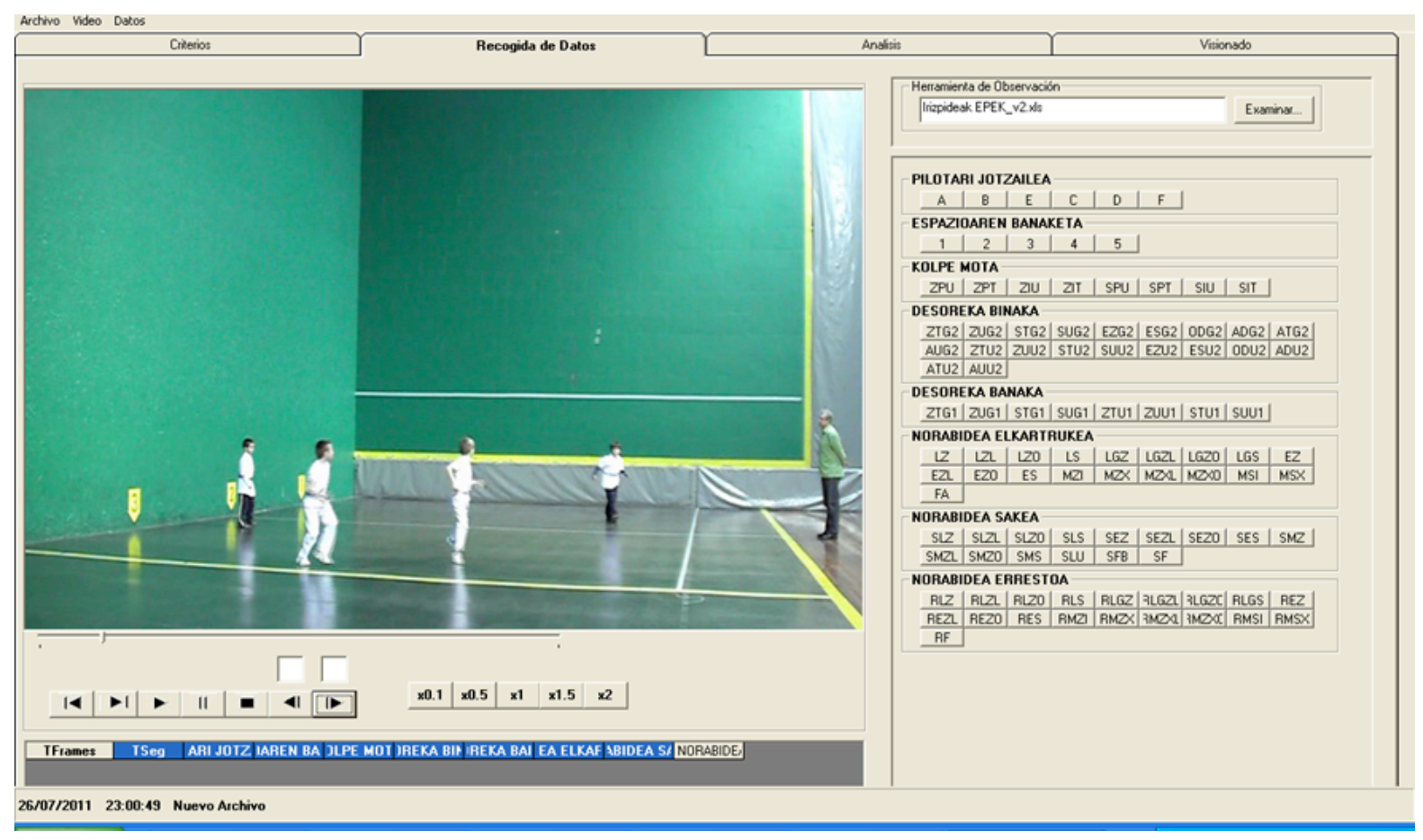

Figura 2. Interface del programa informático MOTS (Castellano y col., 2008). Pilotari jotzailea: pelotari que golpea la pelota; espazioaren banaketa: zonas de golpeo; kolpe mota: tipo de golpe; desoreka binaka: desequilibrio por parejas; desoreka banaka: desequilibrio individual; norabidea elkartrukea: dirección de golpeo de intercambio; norabidea sakea: dirección del saque; norabidea errestoa: dirección del resto.

\section{Procedimiento}

El diseño en el que se plantea este estudio estaría encuadrado en el cuadrante III, atendiendo a los rasgos básicos de: nomotético, puntual y multidimensional (Anguera, BlancoVillaseñor, Hernández-Mendo, y Losada, 2011). De los 35 partidos de pelota a mano escolar grabados durante los cursos escolares 2007/08 y 2008/09, 17 de benjamines (8-10 años) y 18 de alevines, se desecharon 4 partidos (uno de benjamines y tres de alevines) por no estar completos o no cumplir con los mínimos requisitos (falta de observabilidad durante alguna parte del partido o retención del partido por causas ajenas al mismo). Por cada categoría se escogieron seis partidos al azar. Para comprobar la homogeneidad de los seis partidos, en cada una de las categorías (benjamín y alevín), se llevó a cabo un estudio G con un modelo de tres facetas (categoría, partido y categorías del instrumento). Para el plan de medida categorías del instrumento/categorías*partido los coeficientes de generalizabilidad absoluto y relativo fueron próximos a la unidad $(\xi \rho 2(\delta)=0,978$ y $\xi \rho 2(\Delta)=0,976)$, lo que reveló una precisión de generalización de los partidos por categoría óptima, es decir, los partidos fueron similares dentro de cada categoría.

Los doce partidos fueron grabados con una velocidad de obturación de 1/50 seg en ocho localidades distintas, a través de una cámara fija ubicada en el cuadro 7 (24,5 metros) a dos metros de la línea de contracancha. A continuación se convirtieron al formato avi. A partir de la herramienta de observación diseñada (EBSIS.e), el registro de los datos se realizó de manera continua en sesiones de hora y media. Atendiendo a la tipología de los datos, los diferentes criterios que configuran la herramienta de observación se registraron de forma simultánea, anotando aspectos del jugador que golpea la pelota y de los que están en situación de espera. Previo al registro cinco pares de observadores siguieron un plan de entrenamiento de cuatro semanas (30 horas), a partir del protocolo de observación empleado en un estudio previo (Usabiaga y col., 2013). Al tratarse de un deporte donde el vencedor se resuelve con 
un marcador a puntuación límite, los partidos tuvieron diferente ocurrencia de golpes (en cada tanto y en cada partido), por este motivo se procedió a la normalizaron de los registros convirtiéndose los valores absolutos en relativos, medidos como \%, a cada uno de los criterios que componen la herramienta de observación.

\section{Análisis estadísticos}

La media y desviación estándar (DS) de cada variable o categoría fueron analizadas para cuantificar la acción de juego de los escolares durante los 12 partidos, diferenciando las categorías de benjamín y alevín. La comparación de medias se llevó a cabo mediante el test no paramétrico de $U$ de Mann-Whitney, con corrección de Bonferroni, tomando como grupo de comparación las dos categorías, y como variables dependientes las categorías de cada criterio que configura el EBSIS.e. El tamaño del efecto (TE) fue también calculado (Hopkins, 2000) para las variables que resultaron significativas, considerándose que valores inferiores a 0,2 indican un efecto de pequeño tamaño, 0,5 de magnitud media y 0,8 indica un efecto de alta magnitud. El nivel de significación admitido fue de $p<0,05$.

\section{Resultados}

De un total de 12.040 registros (2.408 golpes), cinco registros (jugador que golpea la pelota, zona donde ejecuta el golpe, tipo de golpe, la ubicación de los adversarios respecto al jugador que realiza el golpe y dirección del golpe) por cada golpe codificado, 1.107 (5.535 registros) fueron realizados por benjamines y 1.301 (6.505 registros) por alevines. Como se observa en la tabla 1, no se han encontrado diferencias significativas respecto a las zonas del espacio de juego donde golpean la pelota. En las dos categorías los escolares golpearon la pelota en zonas próximas al frontis y pegados a la pared izquierda (zonas 1, 2 y 4), siendo mínimo el uso de los otros dos espacios de la cancha. Más de la mitad de los golpes se ejecutan entre la línea central longitudinal de la cancha y la pared izquierda, y respecto a la proximidad a la pared frontal, lo hacen cerca del frontis $(26,1 \pm 8,9 \%$ los benjamines y $23,2 \pm 9,0 \%$ los alevines). La relación con las zonas más alejadas del frontis y cercanas al ancho es mínima.

En referencia al tipo de golpe (Tabla 1), los participantes de las dos categorías golpean, sobre todo, con la mano derecha y después de que la pelota haya botado en el suelo $(75,7 \pm 5,5 \%$ los benjamines y 73,0 $\pm 10,0 \%$ los alevines). Las únicas diferencias significativas respecto al tipo de golpe se han encontrado en los de derecha y antes de que la pelota bote en el suelo, suponiendo un mayor número en los alevines $(1,0 \pm 0,4 \%)$, en comparación a los benjamines $(0,2 \pm 0,3 \%)[\mathrm{U}=3,00 ; \mathrm{p}=0,014$ y $\mathrm{TE}=-0,75]$. Los golpes de derecha y de aire no llegan al 2 $\%$ de la suma total y los golpes a bote, de derecha e izquierda, suponen el $98 \%$ restante. 
Usabiaga, O. y Castellano, J. (2014). Uso estratégico del espacio en categorías de formación de pelota vasca. RICYDE. Revista internacional de ciencias del deporte, 36(10), 109-122

http://dx.doi.org/10.5232/ricyde2014.03602

Tabla 1. Valores relativos por partido $(\%)$ en los criterios de las dos categorías analizadas $(* \mathrm{p}<0,05)$.

\begin{tabular}{|c|c|c|c|}
\hline Criterios & Categorías & Benjamín & Alevín \\
\hline \multirow{4}{*}{ Zona de golpe } & Zona 1 & $36,0 \pm 7,3$ & $29,8 \pm 4,8$ \\
\cline { 2 - 4 } & Zona 2 & $31,9 \pm 5,3$ & $40,1 \pm 5,1$ \\
\cline { 2 - 4 } & Zona 3 & $1,8 \pm 2,1$ & $2,0 \pm 1,9$ \\
\cline { 2 - 4 } & Zona 4 & $26,1 \pm 8,9$ & $23,2 \pm 9,0$ \\
\hline \multirow{4}{*}{ Tipo de golpe } & Zona 5 & $4,1 \pm 2,7$ & $5,7 \pm 2,3$ \\
\cline { 2 - 4 } & Izquierda bote & $24,0 \pm 5,4$ & $25,6 \pm 9,6$ \\
\cline { 2 - 4 } & Izquierda aire & $0,0 \pm 0,0$ & $0,3 \pm 0,8$ \\
\cline { 2 - 4 } & Derecha bote & $75,7 \pm 5,5$ & $73,0 \pm 10,0$ \\
\hline \multirow{3}{*}{ Desequilibrio adversarios 2:2 } & Derecha aire* & $0,2 \pm 0,3$ & $1,0 \pm 0,4$ \\
\cline { 2 - 4 } & En la izquierda & $33,0 \pm 5,1$ & $24,2 \pm 8,8$ \\
\cline { 2 - 4 } & En la derecha & $35,4 \pm 4,3$ & $39,6 \pm 3,3$ \\
\hline \multirow{3}{*}{ Desequilibrio adversario 1:1 } & En los dos lados & $31,6 \pm 4,5$ & $36,7 \pm 8,2$ \\
\cline { 2 - 4 } & En la izquierda* & $51,5 \pm 5,4$ & $46,5 \pm 2,7$ \\
\hline \multirow{3}{*}{} & En la derecha* & $48,5 \pm 5,4$ & $53,5 \pm 2,7$ \\
\hline \multirow{3}{*}{ Dirección del saque } & Saque largo & $6,5 \pm 7,5$ & $11,8 \pm 11,7$ \\
\cline { 2 - 4 } & Saque mediano izquierda & $71,2 \pm 10,0$ & $76,8 \pm 14,3$ \\
\cline { 2 - 4 } & Saque mediano derecha & $4,7 \pm 3,5$ & $5,3 \pm 4,5$ \\
\cline { 2 - 4 } & Saque corto & $11,0 \pm 12,7$ & $1,6 \pm 1,9$ \\
\cline { 2 - 4 } & Saque fuera del subespacio & $6,6 \pm 3,3$ & $4,5 \pm 0,0$ \\
\hline
\end{tabular}

Durante los duelos por parejas los jugadores benjamines y alevines se posicionan de forma similar cuando no golpean la pelota, ubicándose a la izquierda, a la derecha y a cada uno de los dos lados del jugador adversario que golpea la pelota. En cambio, cuando juegan duelos individuales se han observado diferencias significativas entre las dos categorías respecto a la ubicación espacial en situación de espera (Tabla 1). Los benjamines se ubican más a la izquierda del oponente que golpea la pelota $(51,5 \pm 5,4 \%)$ y los alevines tienden a posicionarse a la derecha del adversario que golpea la pelota $(53,5 \pm 2,7 \%)[\mathrm{U}=5,00 ; \mathrm{p}=$ $0,037$ y $\mathrm{TE}=-0,22]$. 


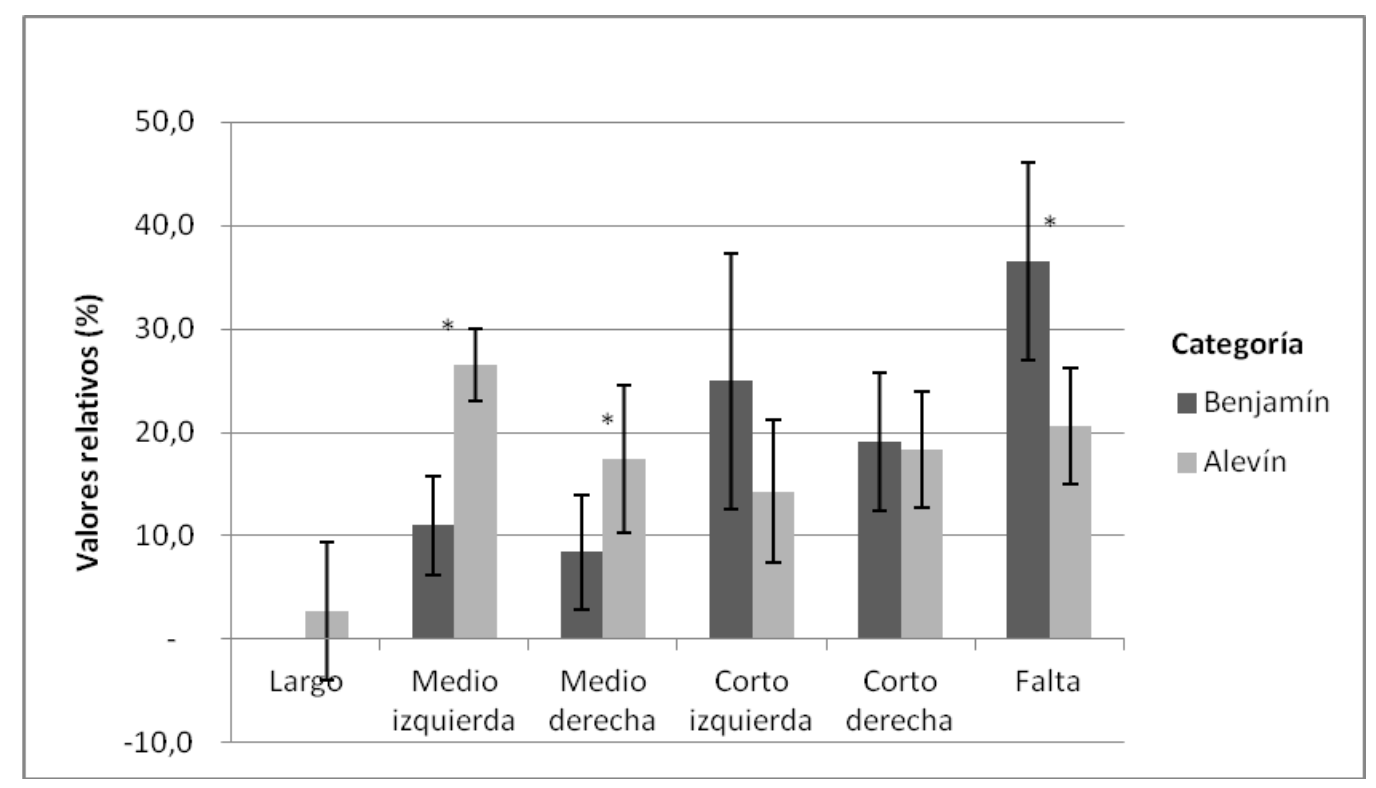

Figura 3. Valores relativos por partido (\%) del criterio dirección del resto de las dos categorías analizadas $(* \mathrm{p}<0,05)$.

Respecto a la dirección del saque, se han agrupado todas las situaciones en las que no continúa el juego (la pasa o media falta, la primera falta de saque y la falta de saque). Los participantes escolares de las dos categorías dirigen los saques a zonas similares del espacio de juego, siendo mayormente a distancias medias y a la izquierda $(71,2 \pm 10,0 \%$ los benjamines y $76,8 \pm 14,3 \%$ los alevines). Respecto a la dirección del resto (Figura 3), se encontraron diferencias significativas en los saques a distancia media a la izquierda $(11,0$ $\pm 4,8 \%$ los benjamines y $26,6 \pm 3,5 \%$ los alevines) $[\mathrm{U}=0,00 ; \mathrm{p}=0,004$ y $\mathrm{TE}=-0,88]$ y a la derecha $(8,4 \pm 5,5 \%$ los benjamines y $17,4 \pm 7,2 \%$ los alevines $)[U=5,00 ; p=0,037$ y $\mathrm{TE}=-$ 0,57], así como en el número de faltas $(36,6 \pm 9,6 \%$ los benjamines y 20,7 $\pm 5,6 \%$ los alevines) $[U=2,00 ; p=0,010$ y $T E=0,71]$. No se encontraron diferencias significativas en los golpes a distancias cortas y largas.

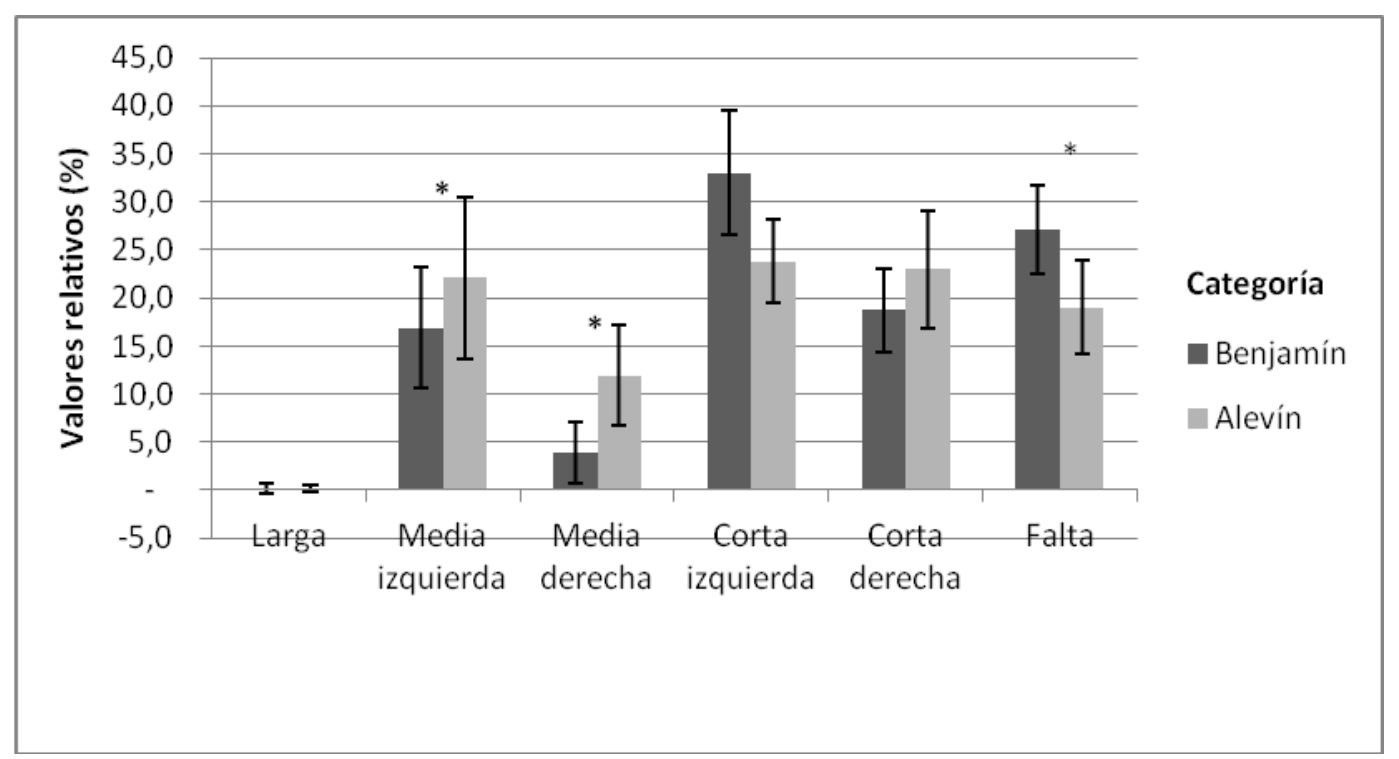

Figura 4. Valores relativos por partido (\%) del criterio dirección del juego de intercambio de las dos categorías analizadas $\left({ }^{*} \mathrm{p}<0,05\right)$. 
Durante el juego de intercambio, los escolares de las dos categorías intercambiaron, sobre todo, golpes próximos al frontis (distancias cortas a la izquierda y derecha). Se han encontrado diferencias significativas respecto a los golpes a media distancia (Figura 4), tanto a la izquierda $(16,9 \pm 6,2 \%$ los benjamines y $22,1 \pm 8,4 \%$ los alevines) $[\mathrm{U}=3,00 ; \mathrm{p}=0,016$ y $\mathrm{TE}=-0,33]$, como a la derecha $(4,0 \pm 3,2 \%$ los benjamines y $11,9 \pm 5,2 \%$ los alevines $)$ [U= $5,00 ; \mathrm{p}=0,037$ y $\mathrm{TE}=-0,67]$. El porcentaje de faltas cometidas durante el juego de intercambio es mayor en los benjamines $(27,1 \pm 4,6 \%)$ que en los alevines $(19,0 \pm 4,9 \%)$ [U= $4,00 ; p=0,026$ y $\mathrm{TE}=0,64]$. La presencia de golpes de larga distancia es mínima en las dos categorías.

\section{Discusión}

En el presente estudio se ha llevado a cabo el análisis y la comparación del uso estratégico del espacio durante el momento del golpe y en situación de espera de los escolares benjamines y alevines que practican pelota a mano dentro del programa de deporte escolar de Gipuzkoa. A partir de la herramienta de observación empleada, se puede interpretar que no existieron diferencias significativas entre benjamines y alevines respecto a las zonas de golpe de la pelota, la ubicación o el desequilibrio espacial de los dos jugadores durante los duelos colectivos y la dirección del saque.

Referente a las zonas donde golpean los jugadores, cabe señalar que los participantes de ambas categorías golpean la pelota en áreas próximas a la pared izquierda y a la pared frontal, a diferencia de las modalidades que utilizan pelotas de goma que contienen aire a presión y se golpean con una raqueta, hacen jugar más lejos de la pared principal tanto a jugadores que juegan más cerca de la pared frontal y habitualmente realizan el saque, como a los que juegan más lejos de la pared frontal (Alonso y col., 2007). La colocación espacial de los jugadores cuando están en situación de espera puede estar influenciada por las zonas de golpe de los adversarios, los cuales son similares, así como la ubicación inicial de cada miembro de la pareja, jugador que juega en la derecha (el que realiza el saque) y jugador que juega en la izquierda. A diferencia de los jugadores de frontenis olímpico masculino (Alonso y col., 2007), la poca salida del material y la consecución del golpe de la pelota con la "mano desnuda" pueden influir en el acercamiento de los jugadores a la pared principal. Aunque el peso y diámetro de la pelota sea diferente para cada categoría, el material revestido de cuero realiza un bote similar respecto a la altura máxima y salida (distancia entre el primer y segundo bote). Sería interesante evaluar los efectos de las adaptaciones progresivas de los materiales y reglas en deportes de iniciación, en la línea de otros estudios (Arias, Argudo, y Alonso, 2011; Giménez, Abad, y Robles, 2010).

En referencia al tipo de golpe, tanto los benjamines como los alevines golpean la pelota sobre todo con la derecha y después de que la pelota haya botado en el suelo. Aunque el porcentaje de golpes de aire y de izquierda es superior en jugadores profesionales de pelota a mano (Usabiaga, 2005), no llegan a ejecutar tantos tipos de golpes como los profesionales de escala $i$ corda de la pelota valenciana (Martínez, Pérez, Chinchilla, y Jiménez, 2013). El peso y diámetro de la pelota pueden ser factores que influyen en este aspecto.

Respecto a la dirección del saque, los escolares de las dos categorías realizaron los mismos tipos de saque, intentando que la pelota se acercase o contactase con la pared izquierda a media distancia, al igual que lo hacen en este mismo tipo de instalación jugadores profesionales de pelota a mano (Usabiaga, 2005) o aficionados de frontenis olímpico (Alonso y col., 2011). Estudios llevados a cabo en deportes de raqueta (Hizan, Whipp, y Reid, 2011; Hughes y col., 2002; O'Donogue, 2004) coinciden en que el saque es un indicador de rendimiento a tener en cuenta para conocer la eficacia del resto. Los benjamines y alevines 
realizan diferentes tipos de resto ante los mismos tipos de saque. Cabe destacar el gran número de faltas que cometen los benjamines en el resto, en comparación con los alevines que también realizan un número importante de errores, con una relevancia importante (efecto de tamaño medio). Este dato es relevante en relación a la continuidad del juego, porque unido a las faltas que se cometen durante el saque, casi la mitad de los tantos se terminan en el primer o segundo golpe. El excesivo peso del saque-resto en jugadores alevines y, sobre todo, benjamines, vuelve a indicar que, quizás, deberían modificarse los reglamentos de juego para favorecer la continuidad en el juego. Proponiendo, por ejemplo, variables didácticas en formato de reglas (Arias y col., 2011) que limiten la dirección del saque. El uso estratégico del espacio durante el saque-resto debería ser uno de los contenidos a trabajar en estas etapas iniciales del proceso de enseñanza-aprendizaje (Egibar y col., 2007), dando importancia a la lectura del comportamiento del adversario que saca y proponiendo estrategias de intervención que faciliten a los restadores indicios de profundidad y apertura de la dirección del saque (Zawadzki y Roca, 2010). Es preciso mantener cierta prudencia en esta interpretación, debido a que este estudio se aplicó en el tenis de alto rendimiento, donde se implican competencias expertas muy diferentes (Martínez de Santos, 2009) a las del juego o mini-deporte propuesto en la etapa formativa.

Referente a la dirección del resto y golpes de intercambio, los alevines, en comparación con los benjamines, dirigieron más golpes a media distancia, tanto a la izquierda como a la derecha, no cometiendo tanta cantidad de faltas. Además, el efecto obtenido es relevante para todas estas diferencias, obteniendo magnitudes medias y, sobre todo, altas. Estos datos indican que los alevines fueron capaces de mantener durante mayor número de golpes la pelota en juego, lo que implicó una mayor diversidad del uso estratégico del espacio durante el golpe de la pelota, acercándose un poco más al tipo de juego desplegado por los profesionales de pelota a mano durante los duelos por parejas (Usabiaga, 2005). Asimismo, los alevines efectuaron más golpes de aire que los benjamines, con una relevancia considerable (efecto de tamaño medio), arrimándose para ello a la pared principal. Esta aproximación al frontis se podría asemejar a la subida a red de tenis, que es considerada una de las conductas tácticas de esta disciplina, y es variable en situaciones de oposición (O’Donoghue, 2009). Por otra parte, el menor porcentaje de golpes de intercambio a distancias cortas de los benjamines supone un aumento del juego "corto", que se ve reflejado en el uso espacial sobre el eje transversal (a la izquierda y a la derecha), realizando un juego cruzado, en este caso preferentemente hacia la izquierda. El porcentaje de faltas que cometen los benjamines durante el juego de intercambio parece indicar nuevamente la necesidad de profundizar en las sesiones didácticas de las etapas iniciales del proceso de enseñanzaaprendizaje, respecto a la lectura del adversario (Parlebas, 2001) asociada al uso estratégico del espacio a través del golpe de la pelota. Uno de los aspectos que puede influir en la consecución eficiente del golpe puede ser la ubicación espacial desde la que parten los jugadores a relacionarse con la pelota. Durante los duelos individuales los alevines tienden a posicionarse más a la derecha del adversario que golpea la pelota, mientras que los benjamines se posicionan en mayor medida a la izquierda. Las instalaciones oficiales donde se practican este tipo de modalidades de raqueta y muro, contienen una pared frontal, otra a la izquierda y otra posterior o trasera, pero no disponen de una muro en la parte derecha. Teniendo en cuenta esta estructuración, existe mayor probabilidad de alejar la pelota del virtual eje central de la cancha (ubicada a cinco metros de la pared izquierda y de la línea de contracancha) hacia la derecha. El recorrido posterior al bote de la pelota no se verá limitado por ninguna barrera (pared o muro), por lo que sería más probable llegar a golpear cualquier pelota si los jugadores cuando están en situación de espera se ubicasen a la derecha del jugador que golpea la pelota. Esta suposición se refuerza si observamos partidos de pelotaris 
profesionales de esta misma disciplina (Usabiaga, 2005).

Los resultados obtenidos en este estudio pueden ayudar a mejorar el proceso de enseñanzaaprendizaje de la pelota a mano en etapas de formación, proponiendo tareas que respondan a las necesidades (tipo de juego) de los escolares de estas edades. "Los alumnos de los centros escolares no son «deportistas de élite bajitos»" (Méndez-Giménez y Fernández-Río, J., 2011), es necesario analizar su juego y la evolución del mismo en las diferentes etapas de formación. Este mismo tipo de análisis ha sido empleado con éxito en el fútbol de formación (Arana, Lapresa, Anguera y Garzón, 2012; Echeazarra, Castellano, y Usabiaga, 2013), pero es necesario que se lleven a cabo más estudios para conocer los efectos que a posteriori puedan generar las tareas propuestas en el juego de los escolares.

De entre las limitaciones del estudio podría incluirse la necesidad de registrar el tipo de material (pelota) que se utiliza en las dos categorías estudiadas. Este estudio podría servir de punto de partida para futuros trabajos, donde se llevasen a cabo análisis de patrones conductuales (Anguera y col., 2011) que nos indicasen la tendencia del flujo conductual durante momentos tan cruciales para la continuidad del juego como el saque-resto, y nos ayudasen a interpretar y comprender mejor el gran porcentaje de faltas que cometen los jugadores de estas categorías.

\section{Conclusiones}

El uso estratégico del espacio de jugadores benjamines y alevines de pelota a mano varía en algunos indicadores de juego como la ubicación espacial del jugador en espera respecto al que golpea la pelota, la dirección del resto y la trayectoria del golpeo de intercambio. Los alevines, en comparación a los benjamines, llevan a cabo más golpes de derecha y aire, se posicionan más a la derecha del jugador que golpea la pelota durante los duelos individuales, dirigen la pelota a distancias medias y cometen menos faltas tanto en el resto del saque como en el juego de intercambio, dando mayor continuidad al juego. Estos indicadores de juego pueden ser útiles en las etapas formativas de la pelota a mano en edad escolar, y pueden aportar al educador/entrenador o el propio jugador información relevante para tomar decisiones en torno al proceso de enseñanza-aprendizaje de dichas disciplinas.

\section{Agradecimientos}

Este trabajo forma parte de la investigación Observación de la interacción en deporte y actividad física: Avances técnicos y metodológicos en registros automatizados cualitativos-cuantitativos, que ha sido subvencionado por la Secretaría de Estado de Investigación, Desarrollo e Innovación del Ministerio de Economía y Competitividad [DEP2012-32124], durante el trienio 2012-2015. Queremos agradecer a los técnicos de la Federación Guipuzcoana de Pelota Vasca toda la ayuda prestada para llevar a cabo el presente estudio. 
Usabiaga, O. y Castellano, J. (2014). Uso estratégico del espacio en categorías de formación de pelota vasca.

\section{Referencias}

Alonso, J. I. (2004). Análisis de la estrategia motriz en el frontenis olímpico. Tesis doctoral: Universidad Católica San Antonio de Murcia.

Alonso, J. I., y Argudo, F. (2011). Análisis notacional informatizado del rendimiento del saque en frontenis olímpico. Revista Internacional de Medicina y Ciencias de la Actividad Física y del Deporte, 11(42), 421-439.

Alonso, J. I., y Argudo, F. (2002, octubre). Influencia de la modalidad técnica de golpeo en el saque sobre la posición del resto y su ejecución. Estudio práxico sobre el frontenis olímpico. Comunicación presentada en el $V$ Congreso Ciencias del Deporte, la Actividad Física y la Recreación. Lérida: INEFC Lleida.

Alonso, J. I., y Argudo, F. (2007). Utilización estratégica del espacio como indicador de rendimiento en el frontenis olímpico masculino. Motricidad. European Journal of Human Movement, 19, 77-95.

Anguera, M. T.; Blanco-Villaseñor, A.; Hernández-Mendo, A., y Losada, J. L. (2011). Diseños observacionales: ajuste y aplicación en psicología del deporte. Cuadernos de Psicología del Deporte, 11(2), 63-76.

Anguera, M. T., y Blanco, A. (2003). Registro y codificación en el comportamiento deportivo. En A. Hernández Mendo (Ed.), Metodología. Buenos Aires: Argentina.

Arana, J.; Lapresa, D.; Anguera, M. T., \& Garzón, B. (2012). Adapting football to the child: an application of the logistic regression model in observational methodology. Quality \& Quantity, 47(6), 3473-3480. http://dx.doi.org/10.1007/s11135-012-9734-z

Araújo, D. (2007). Promoting ecologies where performers exhibit expert interactions. International Journal of Sport Psychology, 38, 73-77

Arias, J. L.; Argudo, F. M., y Alonso, J. I. (2011). Las reglas como variables didácticas. Ejemplo en baloncesto de formación. Revista Internacional de Medicina y Ciencias de la Actividad Física y del Deporte, 11(43), 491-512.

Bakeman, R., y Quera, V. (1996). Análisis de la interacción. Análisis secuencial con SDIS y GSEQ. Madrid: RA-MA [http://www.ub.es/comporta/sg.htm]. (Consultado 20 de febrero de 2013)

Brown, E., \& O'Donoghue, P. G. (2008). Gender and Surface effect on elite tennis strategy. Coaching and Sports Science Review, 46, 9-11. Online. Disponible en: <http://www.itftennis.com/shared/medialibrary/pdf/original/IO_38643_original.PD> (Consultado el 20 de febrero de 2013).

Cabello, D. (2000). Análisis de las características del juego en el bádminton de competición. Su aplicación al entrenamiento. Tesis doctoral: Universidad de Granada.

Castellano, J.; Perea, A.; Alday, L., \& Hernández-Mendo, A. (2008). Measuring and Observation Tool in Sports. Behavior Research Methods, 40(3), 898-903. http://dx.doi.org/10.3758/BRM.40.3.898

Echeazarra, I.; Castellano, J., y Usabiaga, O. (2013). Control de calidad del dato de una herramienta observacional en fútbol formación. Revista Iberoamericana de Psicología del Ejercicio y el Deporte, 8(2), 65-80.

Egibar, M.; Etxeberria, J. C.; Gutierrez, I.; Mendizabal, M.; Usabiaga, O., y Zubiaur, H. (2007). El proceso de enseñanza-aprendizaje de pelota a mano. San Sebastián: Diputación Foral de Gipuzkoa.

Federación Guipuzcoana de Pelota Vasca (2008). Deporte escolar: reglamento de mano. San Sebastián: Federación Guipuzcoana de Pelota Vasca. 
Usabiaga, O. y Castellano, J. (2014). Uso estratégico del espacio en categorías de formación de pelota vasca.

Garay, J. O. (2003). Conceptos clave del tenis de dobles. Tesis doctoral: Universidad del País Vasco UPV/EHU.

Gillet, E.; Leroy, D.; Thouvarecq, R., \& Stein, J. F. (2009). A notational analysis of elite tennis serve and serve-return strategies on slow surface. Journal of Strength \& Conditioning Research, 23(2), 532-539. http://dx.doi.org/10.1519/JSC.0b013e31818efe29

Giménez, F. J.; Abad, M. T., y Robles, J. (2010). El proceso de formación del jugador durante la etapa de iniciación deportiva. Apunts. Educación Física y Deporte, 99(1), 47-55.

Gorospe, G. (1999). Observación y análisis en el tenis de individuales: aportaciones del análisis secuencial y de las coordenadas polares. Tesis doctoral: Universidad del País Vasco UPV/EHU.

Hizan, H.; Whipp, P., \& Reid, M. (2011). Comparison of serve and serve return statistics of high performance male and female tennis players from different age-groups. International Journal of Performance Analysis in Sport, 11(2), 365-375.

Hopkins, W. G. (2000). Measures of reliability in sports medicine and science. Sports Medicine, 30(1), 1-15. http://dx.doi.org/10.2165/00007256-200030010-00001

Hughes, M.D.; Hughes, M.T., \& Behan, H. (2009). Computerized notational analysis and performance profiling in racket sports. En Lees, Cabello \& Torres (eds). Science and Racket Sports IV (pp. 187-196), London: Routledge.

Hughes, M.; Hughes, M. T., \& Behan, H. (2007). The evolution of computerized notational analysis through the example of racket sports. International Journal of Sports Science and Engineering, 11(1), 3-28.

Hughes, M. (1998). The application of notational analysis to racket sports. En Lees, Maynard, Hughes \& Reilly (eds.), Science and Racket Sports, 2 (pp. 211-220), London: $E$ and FN Spon, London.

Hughes, M., \& Barlett, R. (2002). The use of performance indicators in performance analysis. Journal of Sports Sciences, 20, 739-754. http://dx.doi.org/10.1080/026404102320675602

Hughes, M., \& Franks, I. M. (2008). The essentials of performance analysis: an introduction. London: Routledge.

Loffing, F.; Hagemann, N., \& Strauss, B. (2009). The serve in professional men's tennis: Effects of players' handedness. International Journal of Performance Analysis in Sport $9(2), 255-274$

Martínez de Santos, R. (2009). Principios y elementos de organización de la cantera de un equipo de fútbol. Acción Motriz, 2, 61-77.

Martínez, J. A.; Pérez, J. A.; Chinchilla, J. J., y Jiménez, J. M. (2013). Estudio sobre la utilización de gestos técnicos en pelota valenciana en jugadores profesionales. Retos. Nuevas tendencias en Educación Física, Deportes y Recreación, 23(1), 64-66.

Méndez-Giménez, A., y Fernández-Río, J. (2011). Análisis y modificación de los juegos y deportes tradicionales para su adecuada aplicación en el ámbito educativo. Retos. Nuevas tendencias en Educación Física, Deportes y Recreación, 19(1), 54-58.

O'Donoghue, P. G. (2009). Interacting Performances Theory. International Journal of Performance Analysis of Sport, 9, 26-46.

O'Donoghue, P. G. (2010). Research methods for sports performance analysis. London: Routledge. 
O'Donoghue, P. (2004). Match analysis in racket sports. En A. Lees, F. Kahn e I. Maynard (Eds.), Science and racket sports, III (pp. 155-162). London: E \& FN Spon.

Ramos, F. J.; Hernández-Mendo, A.; Pastrana, J. L., y Blanco-Villaseñor, A. (2012). SAGT: Software para la Aplicación de la Teoría de la Generalizabilidad. Proyecto fin de carrera para la titulación: Ingeniería Técnica en Informática de Gestión de la Escuela Técnica Superior de Ingeniería Informática. Universidad de Málaga (España). (Registro Safe Creative Código: 1209202373502 Fecha 20-sep-2012 18:03 UTC).

Rivas, F.; Jaffaye, G., y Cabello, D. (2001). Rol de la información visual en la formación del jugador de bádminton: los currículums informacionales. En IAD (Ed.), IV Congreso Mundial de Bádminton (pp. 327-343). Málaga: Instituto Andaluz del Deporte.

Ruíz, G. (1996). Análisis praxiológico de la estructura del tenis. Comparación de las acciones de juego en la modalidad singles y dobles masculina sobre superficie de tierra batida. Tesis doctoral: Universidad de Las Palmas de Gran Canaria.

Unierzyski, P., \& Wieczorek, A. (2004). Comparison of tactical solutions and game patterns in the finals of two Grand Slam tournaments in tennis. En A. Lees, J. Kahn e I. Maynard (eds.), Science and Racket Sports, 3 (pp. 169-174). Londres: Routledge.

Usabiaga, O. (2005). Evaluación de la acción de juego de la pelota vasca: aplicación en mano parejas. Tesis doctoral: Universidad del País Vasco.

Usabiaga, O.; Castellano, J.; Blanco-Villaseñor, A., y Casamichana, D. (2013). La Teoría de la Generalizabilidad en las primeras fases del método observacional aplicado en el ámbito de la iniciación deportiva: calidad del dato y estimación de la muestra. Revista de Psicología del Deporte, 22(1), 103-109.

Usabiaga, O. y Castellano, J. (2011). Adaptación de la herramienta de observación de la pelota a mano EBSIS para el ámbito formativo. Lecturas: Educación Física y Deportes, 16(162) [http://www.efdeportes.com/efd162/observacion-de-la-pelota-amano-ebsis.htm] (Consultado 20 de febrero de 2013)

Ureña, A. (2003). De las técnicas de observación a la metodología observacional en el deporte. Análisis de tres perspectivas prácticas: investigación, enseñanza y rendimiento. En FACCAFD, Novedades en Ciencias de la Actividad Física y del Deporte, (pp. 43-72). Reprografía Digital Granada.

Vuckovic, G.; James, N., Hughes, M.; Murray, S., Sporis, G., \& Pers, J. (2013). The effect of court location and available time on the tactical shot selection of elite squash players. Journal of Sports Science \& Medicine, 12(1), 66-73.

Zawadzki, P., y Roca, J. (2010). Un estudio sobre indicios perceptivos para el resto en el servicio de tenis. Revista de Psicología del Deporte, 19(1), 59-71. 